\title{
Biological Pest Control Based on Tensor Product Transformation Method
}

\section{Arsit Boonyaprapasorn ${ }^{1}$, Suwat Kuntanapreeda ${ }^{2}$, Teerawat Sangpet $^{2}$, Parinya Sa Ngiamsunthorn ${ }^{3}$ and Eakkachai Pengwang ${ }^{1}$}

\author{
${ }^{1}$ Institute of Field Robotics (FIBO), King Mongkut's University of Technology \\ Thonburi, 126 Pracha Uthit Rd., Bang Mod, Thung Khru, Bangkok 10140, \\ Thailand \\ ${ }^{2}$ Department of Mechanical and Aerospace Engineering, King Mongkut's \\ University of Technology North Bangkok, 1518 Pracharat 1 Rd., Wongsawang, \\ Bangsue, Bangkok 10800, Thailand \\ ${ }^{3}$ Department of Mathematics, Faculty of Science, King Mongkut's University of \\ Technology Thonburi, 126 Pracha Uthit Rd., Bang Mod, Thung Khru, Bangkok \\ 10140, Thailand \\ e-mails: arsit.b@mail.kmutt.ac.th,suwat.k@eng.kmutnb.ac.th, \\ teerawat.s@eng.kmutnb.ac.th, parinya.san@kmutt.ac.th, \\ eakkachai@fibo.kmutt.ac.th
}

\footnotetext{
Abstract: Pest management, based on biological control, has drawn attention from several research groups, due to the exclusion of chemical pesticides, which have debilitating outcomes, both on the environment and human health. Biological pest control policies have been determined using the model-based control approach. In this study, the tensor product model transformation (TPMT) was applied to model the nonlinear dynamic of the biological pest control system. Consequently, the feedback control law representing the biological pest control policy was synthesized based on LMI. Under the designed controller, the pest population was regulated based on the desired level. The simulation of the biological pest control system was presented to confirm the performance of the designed control law. It is evident, that the feedback control method based on TPMT can be employed appropriately, in this application.
}

Keywords: tensor product model transformation method; biological control; pest management; feedback control 


\section{Introduction}

Based on the biological control, pest population was regulated to the desired level through the use of natural enemies, for example, pathogens, parasitoids, predators, antagonists, etc. [1-3]. The biological control can be implemented by three main approaches such as conservation, introduction and augmentation of natural enemies [1-4]. The conservation of natural enemies is applied when the local natural enemies exist in the target area. The introduction approach is employed when local natural enemies cannot handle invasions of nonnative pests. The augmentation is implemented through either the inoculative or the inundation releases depending on the effectiveness in reproducing the augmented natural enemies [1-4]. Several studies and applications of the biological pest control can be found in [1-6].

In general, the dynamic of biological systems can be described by the deterministic models representing the dynamic of ecosystems and epidemic systems as Lotka-Volterra model and compartmental models, respectively [4, 612]. The Lotka-Volterra model is a suitable model for the case of applying natural enemies to control pest population by considering pests as preys and natural enemies as predators.

Using Lotka-Volterra models brings about significant benefits for the attempts to further conduct the dynamic analysis and to determine control policies for regulating or maintaining pest population to be within the economic injury level (EIL) [4, 6, 7-17]. Typically, determination of the biological control policy based on mathematical models can be carried out by using optimal control according to Pontryagin's maximum principle [6, 13-14]. Applications of optimal control for pest management are presented in [6, 13-14]. Rafikov et al. [13-14] employed the Pontryagin's maximum principle to determine the biological control policy for the ecosystem represented by the $n$-dimensional Lotka-Volterra model.

Alternatively, the nonlinear feedback controller design can be utilized for defining the control policies for predator and prey systems [4, 15-18]. In [18], Meza et al. studied various nonlinear feedback control designs for the one-predator one-prey Lotka-Volterra system. Rafikov et al. [4] developed the mathematical model representing the relationship between a group of pests and a group of predators for the biological pest control system. In their work, the control policy was determined based on the LQR controller. Peubla et al. [15] applied the sliding mode control to define the control policy for a general pest control system with the case study of a one-pest and one-predator model. Recently, Peubla et al. [16] employed the modelling error compensation (MEC) method for the biological pest control for a class of the biological control system. Boonyaprapasorn et al. [17] determined the biological pest control based on the synergetic controller design. According to these previous works, the determination of control policies based on a nonlinear feedback control framework for biological processes is feasible, especially, when the system is in the strict feedback form with single input and 
single output. For the case of multiple species of predator and prey systems, with multiple inputs and multiple outputs, defining control policies under the nonlinear feedback control framework may be complicated. Moreover, the design of control law of nonlinear systems is more complicate than that of the linear ones since the standard method of analysis is not available. Complication of synthesis feedback control frame work depends on the structure and characteristics of the nonlinear system, for example, control objective, system order, dimensions of input and outputs, etc. [19-20].

Given the aforementioned complexities, the determination of the control policies under a linear feedback controller design framework is promising compared to existing approaches. One feasible and effective way to approximate these nonlinear ecosystem systems is the tensor product transformation model (TPMT) method. Introduced by Baranyi [21-27], TPMT promised an effective numerical approximation of the nonlinear dynamical system using a convex combination of linear time invariant (LTI) systems. Furthermore, during the transformation process, the high order singular decomposition (HOSVD) is performed such that LMI controller design tool can be executable simultaneously. With the parallel distributed compensation, control design according to LMI can be conducted efficiently based on the TP model [21-27]. As summarized in [24, 26], TP model transformation offers several advantages. For instance, the transformation can be implemented either in the form of equations or soft computing. The numbers of components in the TP model can be defined optimally by balancing between complexity and approximation error. In addition, the order of components is determined based on their relevance. The weighting functions of the TP transformation model can be easily determined under required constraints. Additionally, in the case of the Pseudo TP model transformation, the transformation can be achieved based on predefined weighting functions. The TP model transformation can be used as well to obtain common weighting functions for a set of models.

TPMT has been studied and utilized in the modeling and control of dynamical systems [19, 21-57]. It promises several added benefits in the prevention of overloaded computation [50-51], alleviation of the limitation to apply TPMT caused by different dimensions of inputs and output variables [34], capacities to be implemented under unknown structure [24], improvement of convex hull manipulation to handle the sensitivity problem in LMI-based control design [4653 ] etc. The applications of TPMT in various engineering fields can be found in previous works, such as mechanical engineering [19, 36-38], aerospace engineering [29, 31, 39-41, 48], electrical engineering [42-43], robotics [33, 44] and filter design [45].

The TPMT method has been used as well in the application of biological processes. In previous work by Kovács and Eigner [58], the convex polytopic model of diabetes mellitus was developed and presented based on the TPMT 
approach. Recently, Kovács and Eigner [59] examined the modeling of the tumor growth using this approach.

Since the biological pest control system is a nonlinear control system in the form of Lotka-Volterra model, the system can be expressed as the quasi-linear parameter varying (qLPV) system. Thus, the feedback controller design for this system can be conducted based on the TPMT method [4, 14, 35, 40].

Given the aforementioned effectiveness of TPM-based controller, authors intend to present an alternative and simple approach for defining of biological pest control polies under the linear feedback control framework. Thus, the application of the TPMT feedback controller design scheme to define the biological control policy becomes the main focus of this current work. The considered biological system is represented by a set of Lotka-Volterra equations containing two-pest species and two-predator species.

This paper is organized as follows. In Section 2, the mathematical model of the biological pest control is presented. Then, the TPMT-based controller design aimed to define the biological control policy is described in Section 3. Section 4 presents the simulation results of the biological pest control system under the determined control policy. The last section provides the conclusion of this study.

\section{Mathematical Model of Biological Pest Control}

According to $[4,14]$, the mathematical model of the biological pest control system was developed and represented by Lotka-Volterra system. The Lotka-Volterra model containing two pest species and two natural species is considered in this study. This model is used to describe the interaction between two parasitoids and two caterpillar species.

The growth rates of pest and natural enemy populations depend on the relationship between predators and preys and the biological control as presented in (1) $[4,14]$ :

$$
\left.\begin{array}{l}
\dot{x}_{1}=x_{1}\left(r_{1}-a_{11} x_{1}-a_{12} x_{2}-a_{13} x_{3}-a_{14} x_{4}\right) \\
\dot{x}_{2}=x_{2}\left(r_{2}-a_{21} x_{1}-a_{22} x_{2}-a_{23} x_{3}-a_{24} x_{4}\right) \\
\dot{x}_{3}=x_{3}\left(-r_{3}+a_{31} x_{1}+a_{32} x_{2}\right)+U_{1} \\
\dot{x}_{4}=x_{4}\left(-r_{4}+a_{41} x_{1}+a_{42} x_{2}\right)+U_{2}
\end{array}\right\},
$$

where $x_{1}$ and $x_{2}$ represent the population densities of the first and second caterpillars. The population densities of parasitoids are denoted by $x_{3}$ and $x_{4}$ respectively. The control inputs of the biological control policy corresponding to the growth rate of each parasitoid are defined by $U_{1}$ and $U_{2}$. The reproduction rate or mortality rate of the $i^{\text {th }}$ species is denoted by $r_{i}$, for $i=1, \ldots, 4$ and the 
coefficient $a_{i j}$ represents the rate corresponding to predation, competition and conservation from the interaction between the $i^{\text {th }}$ species and the $j^{\text {th }}$ species for $i, j=1, \ldots, 4$.

The differences between the state variables and control inputs to their corresponding steady values are defined as $y_{1}=x_{1}-x_{1}{ }^{*}, y_{2}=x_{2}-x_{2}{ }^{*}$, $y_{3}=x_{3}-x_{3}{ }^{*}, y_{4}=x_{4}-x_{4}{ }^{*}, u_{1}=U_{1}-u_{1}^{*}$ and $u_{2}=U_{2}-u_{2}{ }^{*}$. The error system can be expressed as (2):

$\dot{\mathbf{y}}=\overline{\mathbf{A}} \mathbf{y}+\mathbf{h}(\mathbf{y})+\mathbf{B u}$,

where $\mathbf{y}=\left[\begin{array}{llll}y_{1} & y_{2} & y_{3} & y_{4}\end{array}\right]^{T}, \mathbf{u}=\left[\begin{array}{ll}u_{1} & u_{2}\end{array}\right]^{T}$,

$$
\overline{\mathbf{A}}=\left[\begin{array}{cccc}
r_{1}-\sum_{j=1}^{4} a_{1 j} x_{j}{ }^{*}-a_{11} x_{1}{ }^{*} & -a_{12} x_{1}^{*} & -a_{13} x_{1}^{*} & -a_{14} x_{1}^{*} \\
-a_{21} x_{2}{ }^{*} & r_{2}-\sum_{j=1}^{4} a_{2 j} x_{j}^{*}-a_{22} x_{2}{ }^{*} & -a_{23} x_{2}{ }^{*} & -a_{24} x_{2}{ }^{*} \\
-a_{31} x_{3}{ }^{*} & -a_{32} x_{3}^{*} & r_{3}-\sum_{j=1}^{4} a_{3 j} x_{j}^{*} & 0 \\
-a_{41} x_{4}{ }^{*} & -a_{42} x_{4}^{*} & 0 & r_{4}-\sum_{j=1}^{4} a_{4 j} x_{j}^{*}
\end{array}\right],
$$

In accordance with [4], the ecosystem (1) can be formulated in the form which is suitable to employ the TPMT. First, the desired steady state of, $x_{1}, x_{2}, x_{3}$ and $x_{4}$ are denoted as $x_{1}{ }^{*}, x_{2}{ }^{*}, x_{3}{ }^{*}$ and $x_{4}{ }^{*}$. The control inputs corresponding to steady state of the system (1) are defined by $u_{1}{ }^{*}$ and $u_{2}{ }^{*}$. Here, $x_{1}{ }^{*}$ and $x_{2}{ }^{*}$ are defined to be below the economic injury level. Then, the values of the corresponding $x_{3}{ }^{*}$, $x_{4}{ }^{*}, u_{1}^{*}$ and $u_{1}^{*}$ can be determined from (3): 


$$
\left.\begin{array}{l}
x_{1}^{*}\left(r_{1}-a_{11} x_{1}^{*}-a_{12} x_{2}^{*}-a_{13} x_{3}^{*}-a_{14} x_{4}^{*}\right)=0 \\
x_{2}^{*}\left(r_{2}-a_{21} x_{1}^{*}-a_{22} x_{2}^{*}-a_{23} x_{3}^{*}-a_{24} x_{4}^{*}\right)=0 \\
x_{3}^{*}\left(-r_{3}+a_{31} x_{1}^{*}+a_{32} x_{2}^{*}\right)+u_{1}^{*}=0 \\
x_{4}^{*}\left(-r_{4}+a_{41} x_{1}^{*}+a_{42} x_{2}^{*}\right)+u_{2}^{*}=0
\end{array}\right\}
$$

Readers can find more information about the mathematical model from previous works by Rafikov et al. [4] and Molter and Rafikov [14].

\section{Tensor Product Transformation-based Controller Design of Biological Pest Control}

In this section, the details about the application of the TPMT-based feedback control are provided. First, conversion of the biological pest control system by using the TPMT is presented in Section 3.1. Then, the detail of feedback controller design is explained in Section 3.2.

\subsection{Tensor Product Model Transformation of Biological Pest Control System}

According to [21-23, 25-26, 31], the state error corresponding to the ecosystem in (2) can be transformed to the convex tensor product (TP) model, which can be conducted as follows.

First, the dynamic error in (2) can be represented in the form of quasi-linear time varying parameters (qLPV) constructed as (4):

$$
\begin{aligned}
\dot{\mathbf{y}} & =\mathbf{A}(\mathbf{p}(t)) \mathbf{y}+\mathbf{B u} \\
& =\left[\begin{array}{ll}
\mathbf{A}(\mathbf{p}(t)) & \mathbf{B}(\mathbf{p}(t))
\end{array}\right]\left[\begin{array}{l}
\mathbf{y} \\
\mathbf{u}
\end{array}\right] \\
\dot{\mathbf{y}} & =\mathbf{S}(\mathbf{p}(t))\left[\begin{array}{l}
\mathbf{y} \\
\mathbf{u}
\end{array}\right],
\end{aligned}
$$

where $\mathbf{S}(\mathbf{p}(t))=\left[\begin{array}{ll}\mathbf{A}(\mathbf{p}(t)) & \mathbf{B}(\mathbf{p}(t))\end{array}\right]$ is the system matrix. Considering $\overline{\mathbf{A}}$ and $\overline{\mathbf{h}}$ in (2), the component of the matrix $\mathbf{A}$ can be selected as (5):

$$
A_{11}=\left(r_{1}-\sum_{j=1}^{4} a_{1 j} x_{j}^{*}-a_{11} x_{1}^{*}\right)-a_{11} p_{1}, A_{12}=-a_{12} x_{1}^{*}-a_{12} p_{1}
$$




$$
\begin{aligned}
& A_{13}=-a_{13} x_{1}^{*}-a_{13} p_{1}, A_{14}=-a_{14} x_{1}^{*}-a_{14} p_{1}, A_{21}=-a_{21} x_{2}^{*}-a_{21} p_{2} \\
& A_{22}=\left(r_{2}-\sum_{j=1}^{4} a_{2 j} x_{j}^{*}-a_{22} x_{2}^{*}\right)-a_{22} p_{2}, A_{23}=-a_{23} x_{2}^{*}-a_{23} p_{2} \\
& A_{24}=-a_{24} x_{2}^{*}-a_{24} p_{2}, A_{31}=-a_{31} x_{3}^{*}, A_{32}=-a_{32} x_{3}^{*} \\
& A_{33}=r_{3}-\sum_{j=1}^{4} a_{3 j} x_{j}^{*}-\left(a_{31} p_{1}+a_{32} p_{2}\right), A_{34}=0, A_{41}=-a_{41} x_{4}^{*} \\
& A_{42}=-a_{42} x_{4}^{*}, A_{43}=0, A_{44}=r_{4}-\sum_{j=1}^{4} a_{4 j} x_{j}^{*}-\left(a_{41} p_{1}+a_{42} p_{2}\right),
\end{aligned}
$$

where $p_{1}(t)=y_{1}(t)$ and $p_{2}(t)=y_{2}(t)$

Then, the TPMT is applied to the qLPV system in (4). The convex combination of $R$ constant linear time invariant (LTI) system representing the qLPV system can be obtained as (6):

$$
\mathbf{S}(\mathbf{p}(t))=\sum_{r=1}^{R} \omega_{r}(\mathbf{p}(t)) \mathbf{S}_{r},
$$

where the weighting function is defined by $\omega_{r}(\mathbf{p}(\mathbf{t}))$ and $\mathbf{S}_{\mathbf{r}}$ is the $r^{\text {th }}$ linear time invariant system denoted as (7):

$$
\mathbf{S}_{\mathbf{r}}=\left[\begin{array}{ll}
\mathbf{A}_{\mathbf{r}} & \mathbf{B}_{\mathbf{r}}
\end{array}\right], r=1,2, \ldots, R .
$$

Therefore, the system in (4) can be expressed as (8):

$$
\dot{\mathbf{y}}=\sum_{r=1}^{R} \omega_{r}(\mathbf{p}(t))\left(\mathbf{A}_{\mathbf{r}}+\mathbf{B}_{\mathbf{r}} \mathbf{u}\right) \text {. }
$$

\subsection{Controller Design}

According to $[4,11]$, the objective control is to regulate the population of the pests to the desired level corresponding to the economic injury level (EIL). Thus, the control inputs need to be designed such that the system in (2) is stable.

The system in (4) is transformed into the TP model in (8). Then, the controller design can be performed according to a parallel distributed compensation (PDC) controller design frame work. The feedback controller is determined as (9):

$$
\mathbf{u}=-\left(\sum_{r=1}^{R} \omega_{r}(\mathbf{p}(t)) \mathbf{K}_{r}\right) \mathbf{y} .
$$

The gain matrices, $\mathbf{K}_{r}$, can be determined by solving the LMIs as shown in (10) and (11) $[21,25]$ : 
$-Y \mathbf{A}_{r}^{T}-\mathbf{A}_{r} Y+\mathbf{M}_{r}^{T} \mathbf{B}_{r}^{T}+\mathbf{B}_{r} \mathbf{M}_{r}>0$ for $r=1, \ldots, R$

and

$$
\begin{aligned}
-\mathbf{Y} \mathbf{A}_{r}^{T}-\mathbf{A}_{r} \mathbf{Y}-\mathbf{Y} \mathbf{A}_{s}^{T}-\mathbf{A}_{s} \mathbf{Y} & \text { for } r \leq s \leq R, \\
& +\mathbf{M}_{s}^{T} \mathbf{B}_{r}^{T}+\mathbf{B}_{r} \mathbf{M}_{s}+\mathbf{M}_{r}^{T} \mathbf{B}_{s}^{T}+\mathbf{B}_{s} \mathbf{M}_{r} \geq 0
\end{aligned}
$$

where $\mathbf{K}_{r}$ is defined as $\mathbf{K}_{r}=\mathbf{M}_{r} \mathbf{Y}^{-1}$. Under the control law in (7), the control system (4) can be stabilized asymptotically. Further details about the TPMT can be found in [21-23, 25-26, 31]. The TP tool program [23] can be employed to determine the TPMT and the feedback control in (9).

\section{Simulations}

The simulation results of the ecosystem representing the biological pest control system manipulated by the designed control law from Section 3 are presented and discussed. Here, the system parameters of (1) were from the previous works [4, 14] as follows: (i) $r_{1}=0.17, r_{2}=0.17, \quad r_{3}=0.119, r_{4}=0.119$, (ii) $a_{11}=0.00017, a_{12}=0.00017, a_{13}=0.0017, a_{21}=0.000255, a_{22}=0.00017$, $a_{23}=0.00017, a_{24}=0.0017, a_{31}=0.00085, a_{32}=0.000085, a_{41}=0.00425$, $a_{42}=0.00425$. The desired values of $x_{1}^{*}$ and $x_{2}{ }^{*}$ were defined as $x_{1}^{*}=9$ and $x_{2}{ }^{*}=9$, which corresponded to the economic injury level. From (3), it yielded $x_{3}^{*}=0.5, x_{4}^{*}=97.7, u_{1}^{*}=0.0553$ and $u_{2}^{*}=4.1522$. The spaces of $p_{1}(t)=y_{1}(t)$ and $p_{2}(t)=y_{2}(t)$ were selected as $[-5,5]$ and $[-5,5]$, respectively.

By using the TP tool program [23] with $100 \times 100$ sampling, the rank of the sampled tensor was found to be 2 on both dimensions. Thus, the system can be exactly represented by 4 vertex systems. Figures 1 and 2 display the obtained weighting functions. By solving the LMI conditions, it resulted in the following four linear feedback gains:

$$
\begin{aligned}
K_{1,1} & =\left[\begin{array}{cccc}
-13.6887 & 402.4572 & 1.1556 & -15.1487 \\
-257.5399 & 82.4338 & 11.7128 & 4.8434
\end{array}\right] \\
K_{2,1} & =\left[\begin{array}{cccc}
-126.2939 & -2.7031 & 5.2014 & 4.1584 \\
96.6865 & -219.2167 & -4.7439 & 4.8572
\end{array}\right] \\
K_{1,2} & =\left[\begin{array}{llll}
-31.1574 & -284.9870 & 0.8807 & 12.0729 \\
250.5103 & -238.4877 & -11.5536 & 0.9592
\end{array}\right]
\end{aligned}
$$


$K_{2,2}=\left[\begin{array}{cccc}-97.7995 & 422.5019 & 4.6144 & -13.3817 \\ -345.1646 & 256.9620 & 15.5817 & 1.2851\end{array}\right]$.

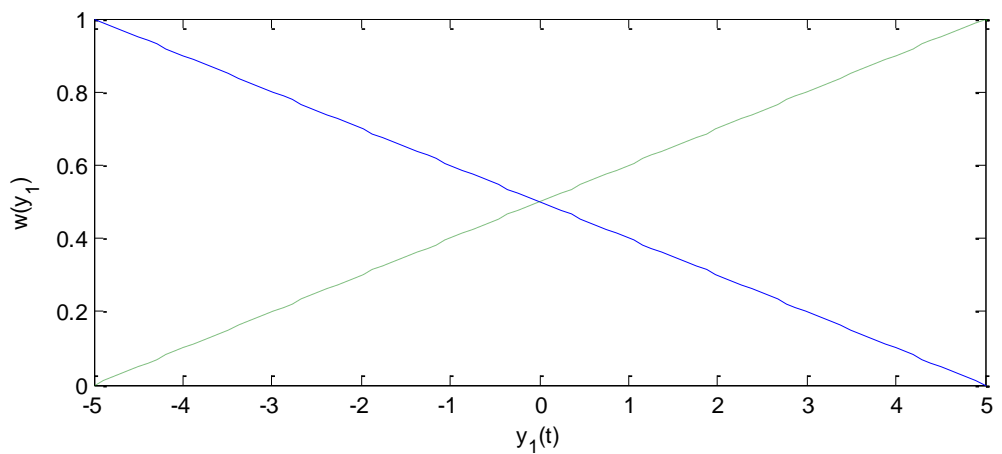

Figure 1

Weighting function on $p_{1}(t)=y_{1}(t)$

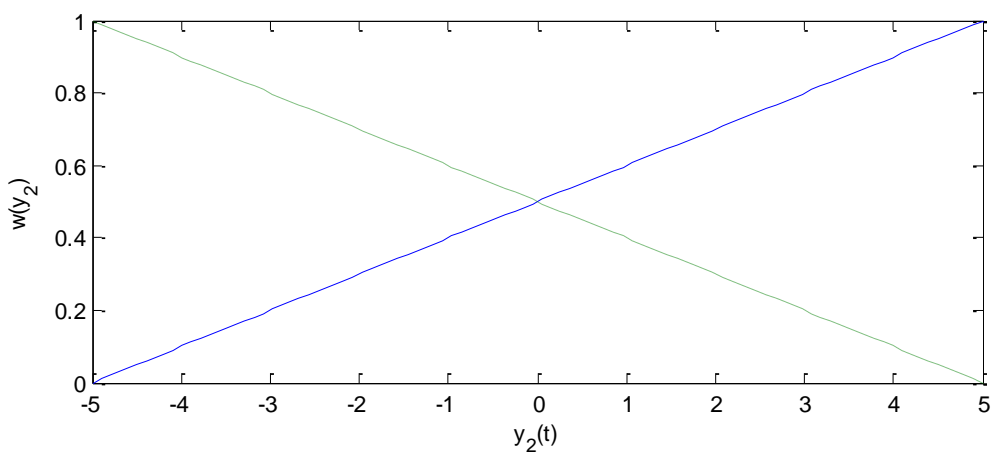

Figure 2

Weighting function on $p_{2}(t)=y_{2}(t)$

The time responses corresponding to the pests (caterpillars) and predators (parasitoids) of the biological control system as manipulated by the feedback control in (9) are presented in Figure 3. The plots of control inputs are shown in Figure 4. It was evident that the time responses of all pest converged to the desired levels as shown in Figure 3. Based on the synthesized control policy, the pest populations converged to the desired level which did not cause the economic damage. 

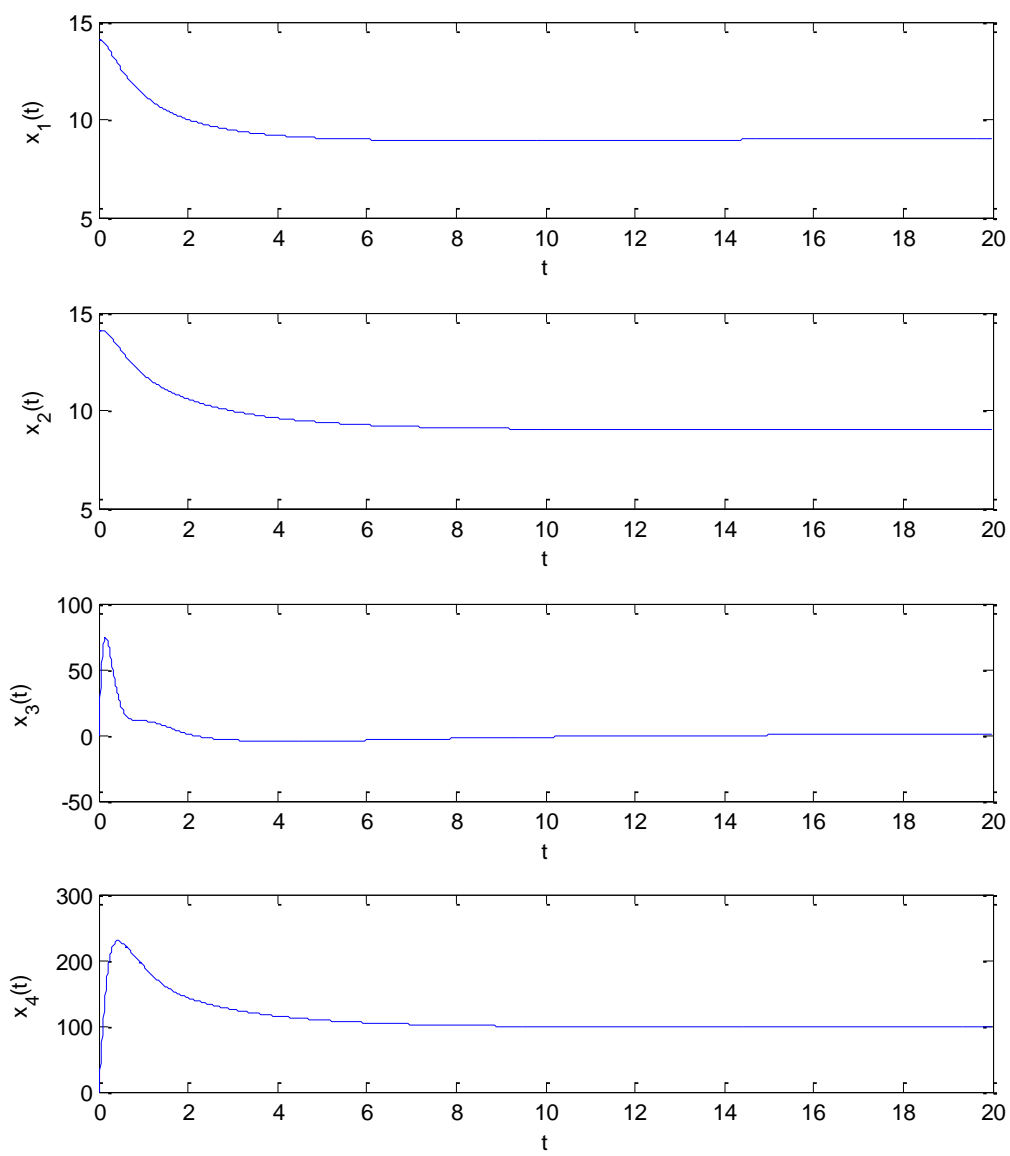

Figure 3

Time response of the Biological pest control system 

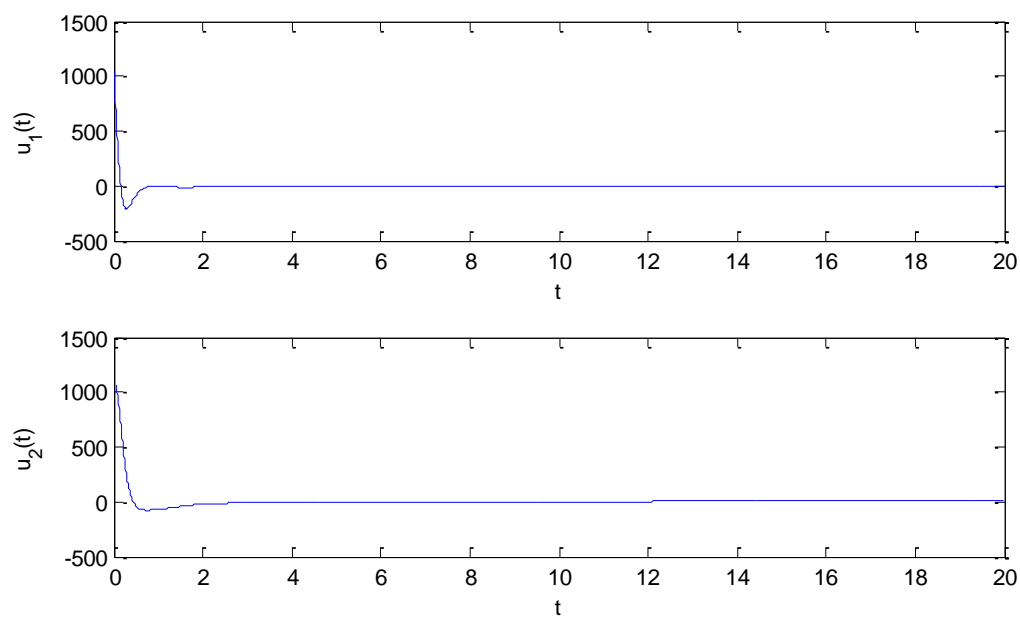

Figure 4

Biological control policy

\section{Conclusion}

From the current findings, the feedback controller design procedure, based on the tensor product model transformation (TPMT), was successfully employed to define control policy for the biological pest control system. According to the determined control policy, the pest populations were regulated at corresponding desired levels. The performance of the control method was confirmed by the simulation. Thus, the TPMT-based feedback control is recommended as an alternative method for determining control policy for biological pest control systems.

\section{Acknowledgement}

This research is supported by the Petchra Pra Jom Klao Ph.D. Research Scholarship from King Mongkut's University of Technology Thonburi.

\section{References}

[1] R. G. Van Driesche and T. S. Bellows Jr.: Biological Control, New York: Chapman \& Hall, Springer US, 1996

[2] R. Van den Bosch, P. S. Messenger and A. P. Gutierrez: An Introduction to Biological Control, New York: Plenum Press, 1982

[3] P. Debach and D. Rosen: Biological Control by Natural Enemies, $2^{\text {nd }}$ Ed., Vol. 8, Cambridge: Cambridge University Press, 1991

[4] M. Rafikov, J. M. Balthazarand and H. F. von Bremen: Mathematical Modeling and Control of Population Systems: Applications in Biological Pest Control, Applied Mathematics and Computation, Vol. 200, Issue 2, 2008, pp. 557-573 
[5] A. Kergunteuil, M. Bakhtiari, L. Formenti, Z. Xiao, E. Defossez and S. Rasmann: Biological Control Beneath the Feet: A Review of Crop Protection against Insect Root Herbivores. Insects, Vol. 7, No. 4, 2016, pp. 70

[6] B.-S. Goh: Management and Analysis of Biological Populations, Vol. 8, Amsterdam: Elsevier Scientific Publishing Company, 1980

[7] H. I. Freedman: Deterministic Mathematical Models in Population Ecology, Vol. 57, New York: Marcel Dekker Incorporated, 1980

[8] A. J. Lotka: Fluctuations in the Abundance of Species Considered Mathematically (with comment by V. Volterra), Nature, Vol. 119, 1927, pp. 12-13

[9] V. Volterra: Fluctuations in the Abundance of the Species Considered Mathematically, Nature, Vol. 118, 1926, pp. 558-560

[10] A. N. Kolmogorov: Sulla Teoria di Volterra della Lotta per l'Esistenza. Giorn. Instituto Ital. Attuari, Vol. 7, 1936, pp. 74-80

[11] S. Tang and R. A. Cheke: Models for Integrated Pest Control and Their Biological Implications, Mathematical Biosciences, Vol. 215, Issue 1, 2008, pp. 115-125

[12] Y. Tan and L. Chen: Modelling Approach for Biological Control of Insect Pest by Releasing Infected Pest, Chaos, Solitons \& Fractals, Vol. 39, Issue 1, 2009, pp. 304-315

[13] M. Rafikov and J. M. Balthazar: Optimal Pest Control Problem in Population Dynamics, Journal of Computational and Applied Mathematics, Vol. 24, No. 1, 2005, pp. 65-81

[14] A. Molter and M. Rafikov: Nonlinear Optimal Control of Population Systems: Applications in Ecosystems, Nonlinear Dynamics, Vol. 76, 2014, pp. 1141-1150

[15] H. Puebla, A. Morales-Diaz and A. V. Pérez: Sliding Mode Control for Biological Pest Control Problems, in Proc. of Congreso Nacional de Control Automático (AMCA 2015), Cuernavaca, Morelos, México, 2015, pp. 201-204

[16] H. Puebla, P. K. Roy, A. Velasco-Perez and M. M. Gonzalez-Brambila: Biological Pest Control Using a Model-Based Robust Feedback, IET Systems Biology, Vol. 12, Issue 6, 2018, pp. 233-240

[17] A. Boonyaprapasorn, P. Sa-Ngiumsunthorn, S. Natsupakpong and S. Laoaroon: Biological Pest Control Using Synergetic Controller with Ant Colony Optimization, in Proc. of the $28^{\text {th }}$ Annual Meeting of the Thai Society for Biotechnology and International Conference, Chiang Mai, Thailand, 2016 
[18] M. E. M. Meza, A. Bhaya and E. Kaszkurewicz: Controller Design Techniques for the Lotka-Volterra Nonlinear System, Sba: Controle \& Automação Sociedade Brasileira de Automatica, Vol. 16, No. 2, 2005

[19] S. John and J. O. Pedro: Neural Network-Based Adaptive Feedback Linearization Control of Antilock Braking System, International Journal of Artificial Intelligence, Vol. 10, 2013, pp. 21-40

[20] S. Oancea, I. Grosu and A. V. Oancea: Biological Control Based on the Synchronization of Lotka-Volterra Systems with Four Competitive Species, Romanian Journal of Biophysics, Vol. 21, No. 1, 2011, pp. 17-26

[21] P. Baranyi: TP Model Transformation as a Way to LMI-based Controller Design, IEEE Transactions on Industrial Electronics, Vol. 51, No. 2, 2004, pp. $387-400$

[22] P. Baranyi and A. R. Várkonyi-Kóczy: TP Transformation Based Dynamic System Modeling for Nonlinear Control, IEEE Transactions on Instrumentation and Measurement, Vol. 54, No. 6, 2005, pp. 2191-2203

[23] S. Nagy, Z. Petres and P. Baranyi: TP Tool - A MATLAB Toolbox for TP Model Transformation, in Proc. of the $8^{\text {th }}$ International Symposium Hungarian Researchers on Computational Intelligence and Informatics (CINTI 2007), Hungary, 2007, pp. 483-495

[24] P. Baranyi: Extracting LPV and qLPV Structures from State-space Functions: A TP Model Transformation Based Framework, IEEE Transactions on Fuzzy Systems, 2019, pp. 1-1

[25] P. Baranyi, Y. Yam and P. Várlaki: Tensor Product Model Transformation in Polytopic Model-based Control, CRC Press, 2014

[26] P. Baranyi: TP-Model Transformation-Based-Control Design Frameworks, Springer International Publishing, 2016

[27] K. Tanaka and H. O. Wang: Fuzzy Control Systems Design and AnalysisA Linear Matrix Inequality Approach. New York: John Wiley \& Sons, Inc., 2001

[28] G. Zhao, D. Wang and Z. Song: A Novel Tensor Product Model Transformation-Based Adaptive Variable Universe of Discourse Controller, Journal of the Franklin Institute, Vol. 353, Issue 17, 2016, pp. 4471-4499

[29] T. Jiang and D. Lin: Tensor Product Model-Based Gain Scheduling of a Missile Autopilot, Transactions of Japan Society for Aeronautical and Space Sciences, Vol. 59, Issue 3, 2016, pp. 142-149

[30] Y. Kan, Z. He and J. Zhao: Tensor Product Model-Based Control Design with Relaxed Stability Conditions for Perching Maneuvers, Acta Polytechnica Hungarica, Vol. 15, No. 3, 2018, pp. 45-61 
[31] S. Kuntanapreeda: Control of Shimmy Vibration in Aircraft Landing Gears Based on Tensor Product Model Transformation and Twisting Sliding Mode Algorithm, in Proc. of the $13^{\text {th }}$ International Scientific-Technical Conference on Electromechanics and Robotics "Zavalishin's Readings"2018, Russia, 2018, MATEC Web of Conferences, Vol. 161, Article No. 02001

[32] S. Kuntanapreeda: Tensor Product Model Transformation Based Control and Synchronization of a Class of Fractional-order Chaotic Systems. Asian Journal of Control, Vol. 17, Issue 2, 2015, pp. 371-380

[33] P. Galambos and P. Baranyi: Representing the Model of Impedance Controlled Robot Interaction with Feedback Delay in Polytopic LPV Form: TP Model Transformation Based Approach, Acta Polytechnica Hungarica, Vol. 10, No. 1, 2013, pp. 139-157

[34] P. Baranyi: Extension of the Multi-TP Model Transformation to Functions with Different Numbers of Variables, Complexity, Vol. 2018, 2018, Article ID 8546976

[35] C. Pozna and R.-E. Precup: An Approach to the Design of Nonlinear StateSpace Control Systems, Studies in Informatics and Control, Vol. 27, No. 1, 2018, pp. 5-14

[36] J. Kuti, P. Galambos and Á. Miklós: Output Feedback Control of a DualExcenter Vibration Actuator via qLPV Model and TP Model Transformation, Asian Journal of Control, Vol. 17, Issue 2, 2015, pp. 432442

[37] J. Matuško, Š. Ileš, F. Kolonić and V. Lešić: Control of 3D Tower Crane Based on Tensor Product Model Transformation with Neural Friction Compensation, Asian Journal of Control, Vol. 17, Issue 2, 2015, pp. 443458

[38] P. Gróf and Y. Yam: Furuta Pendulum - A Tensor Product Model-Based Design Approach Case Study, in Proc. of the 2015 IEEE International Conference on Systems, Man, and Cybernetics (SMC 2015), Hong Kong, 2015, pp. 2620-2625

[39] Z. He, M. Yin and Y. Lu: Tensor Product Model-Based Control of Morphing Aircraft in Transition Process, Proceedings of the Institution of Mechanical Engineers, Part G: Journal of Aerospace Engineering, Vol. 230, Issue 2, 2016, pp. 378-391

[40] H. Du, J. Yan and Y. Fan: A State and Input Constrained Control Method for Air-Breathing Hypersonic Vehicles, Acta Polytechnica Hungarica, Vol. 15, No. 3, 2018, pp. 81-99

[41] B. Takarics, A. Szöllősi and B. Vanek: Tensor Product Type Polytopic LPV Modeling of Aeroelastic Aircraft, in Proc. of 2018 IEEE Aerospace Conference, Big Sky, MT, USA, 2018, pp. 1-10 
[42] A. M. F. Pereira, L. M. S. Vianna, N. A. Keles and V. C. S. Campos: Tensor Product Model Transformation Simplification of Takagi-Sugeno Control and Estimation Laws - An Application to a Thermoelectric Controlled Chamber, Acta Polytechnica Hungarica, Vol. 15, No. 3, 2018, pp. 13-29

[43] Y. Zhou, J. Liu, Y. Li, C. Gan, H. Li and Y. Liu: A Gain Scheduling WideArea Damping Controller for the Efficient Integration of Photovoltaic Plant, IEEE Transactions on Power Systems, Vol. 34, No. 3, 2019, pp. 1703-1715

[44] X. Han, T. Wang and S. Yu: Predictive Control of Mobile Robot Based on Tensor Product Model Transformation, in Proc. of $201729^{\text {th }}$ Chinese Control and Decision Conference (CCDC 2017), Chongqing, China, 2017, pp. $7872-7876$

[45] H. Gong, Y. Yu, L. Zheng, B. Wang, Z. Li, T. Fernando, H. H. C. Iu, X. Liao and X. Liu: Nonlinear Hळ Filtering Based on Tensor Product Model Transformation, IEEE Transactions on Circuits and Systems II: Express Briefs, 2019, pp. 1-1

[46] P. Várkonyi, D. Tikk, P. Korondi and P. Baranyi: A New Algorithm for RNO-INO Type Tensor Product Model Representation, in Proc. of 2005 IEEE International Conference on Intelligent Engineering Systems (INES '05), Cruising on the Mediterranean Sea, Spain, 2005, pp. 263-266

[47] A. Szollosi and P. Baranyi: Influence of the Tensor Product Model Representation of qLPV Models on the Feasibility of Linear Matrix Inequality, Asian Journal of Control, Vol. 18, Issue 4, 2016, pp. 1328-1342

[48] A. Szollosi and P. Baranyi: Improved Control Performance of the 3-DoF Aeroelastic Wing Section: A TP Model Based 2D Parametric Control Performance Optimization, Asian Journal of Control, Vol. 19, Issue 2, 2017, pp. 450-466

[49] A. Szollosi and P. Baranyi: Influence of the Tensor Product Model Representation of qLPV Models on the Feasibility of Linear Matrix Inequality Based Stability Analysis, Asian Journal of Control, Vol. 20, Issue 1, 2018, pp. 531-547

[50] J. Cui, K. Zhang and T. Ma: An Efficient Algorithm for the Tensor Product Model Transformation, International Journal of Control, Automation and Systems, Vol. 14, 2016, pp. 1205-1212

[51] S. Nagy, Z. Petres, P. Baranyi and H. Hashimoto: Computational Relaxed TP Model Transformation: Restricting the Computation to Subspaces of the Dynamic Model, Asian Journal of Control, Vol. 11, Issue 5, 2009, pp. 461475

[52] X. Liu, Y. Yu, Z. Li, H. H. C. Iu and T. Fernando: An Efficient Algorithm for Optimally Reshaping the TP Model Transformation, IEEE Transactions 
on Circuits and Systems II: Express Briefs, Vol. 64, No. 10, 2017, pp. 1187-1191

[53] J. Kuti, P. Galambos and P. Baranyi: Minimal Volume Simplex (MVS) Polytopic Model Generation and Manipulation Methodology for TP Model Transformation, Asian Journal of Control, Vol. 19, Issue 1, 2017, pp. 289301

[54] S. Deng, J. Liu and X. Wang: The Properties of Fuzzy Tensor and Its Application in Multiple Attribute Group Decision Making, IEEE Transactions on Fuzzy Systems, Vol. 27, No. 3, 2019, pp. 589-597

[55] Y. Yu, J. Feng, J. Pan and D. Cheng: Block Decoupling of Boolean Control Networks, IEEE Transactions on Automatic Control, Vol. 64, No. 8, 2019, pp. 3129-3140

[56] Y. Yu, Z. Li, X. Liu, K. Hirota, X. Chen, T. Fernando and H. H. C. Iu: A Nested Tensor Product Model Transformation, IEEE Transactions on Fuzzy Systems, Vol. 27, No. 1, 2019, pp. 1-15

[57] X. Liu, X. Xin, Z. Li and Z. Chen: Near Optimal Control Based on the Tensor-Product Technique, IEEE Transactions on Circuits and Systems II: Express Briefs, Vol. 64, No. 5, 2017, pp. 560-564

[58] L. Kovács and Gy. Eigner: Convex Polytopic Modeling of Diabetes Mellitus: A Tensor Product based approach, in Proc. of 2016 IEEE International Conference on Systems, Man, and Cybernetics (SMC), Budapest, Hungary, 2016, pp. 003393-003398

[59] L. Kovács and Gy. Eigner: Tensor Product Model Transformation Based Parallel Distributed Control of Tumor Growth, Acta Polytechnica Hungarica, Vol. 15, No. 3, 2018, pp. 101-123 